\title{
Thermal Time Constant of Nb Diffusion-Cooled Superconducting Hot-Electron Bolometer Mixers
}

\author{
D. Wilms Floet, J.R. Gao, T. M. Klapwijk, and P. A. J. de Korte
}

\begin{abstract}
We present an experimental study of the intermediate frequency bandwidth of a $\mathrm{Nb}$ diffusion-cooled hotelectron bolometer mixer for different bias voltages. The measurements show that the bandwidth increases with increasing voltage. Analysis of the data reveals that this effect is mainly caused by a decrease of the intrinsic thermal time constant of the mixer and that the effect of electrothermal feedback through the intermediate frequency circuit is small. The results are understood using a qualitative model, which takes into account the different effective diffusion constants in the normal and superconducting domains.
\end{abstract}

Keywords - Superconducting THz detectors, electronic hot spot, thermal time constant, bolometers.

\section{INTRODUCTION}

$\mathrm{T}$ HE STRONG NEED for sensitive heterodyne mixers at terahertz frequencies is an important stimulus for the ongoing development of hot-electron bolometer (HEB) mixers. Besides the rapid progress on the experimental side, new light has been shed on the physical mechanisms that govern the HEB in heterodyne operation. Classically, the HEB is described as a lumped element, making use of the steep rise of the direct-current $(\mathrm{dc})$ resistance $R$ as a function of the temperature $T$ close to the critical temperature $T_{c}[1],[2],[3]$. The resistive transition $R(T)$ is usually measured in the zero-bias limit and without applied radiofrequency (rf) radiation. In a heterodyne mixing situation, i.e. with high dc and $\mathrm{rf}$ dissipation and at a bath temperature $T_{b}$ below $T_{c}$, the $R(T)$ is no longer relevant[4] and the presence of a temperature profile in the microbridge has to be taken into account[5]. A new concept has been introduced in which heterodyne mixing in HEBs is described in terms of an electronic hotspot (EHS) of which the length $L_{h}$ oscillates at the intermediate frequency (IF) [6], [7]. Recently, a similar physical model was introduced, which also takes into account the temperature profile, but assumes a different temperature dependence of the local resistivity [8].

\section{Thermal Time constant of HeBs}

The IF gain bandwidth of a HEB is defined as the frequency where the gain has dropped $3 \mathrm{~dB}$ below its zero frequency value (roll-off frequency $f_{-3 d B}$ ), and is physically

Manuscript received September 15,2000 . This work is financially supported by the Stichting voor Technische Wetenschappen, which is part of the Nederlandse Organisatie voor Wetenschappelijk Onderzoek and, by ESA under contract no. 11738/95/NL/PB. D. Wilms Floet and T. M. Klapwijk are with the Department of Applied Physics and DIMES, Delft University of Technology, Lorentzweg 1, $2628 \mathrm{CJ}$ Delft, The Netherlands, J. R. Gao and P. A. J. de Korte are with the Space Research Organization of the Netherlands, Sorbonnelaan 2 3584 CA Utrecht, The Netherlands. Corresponding authors' E-mail j.r.gao@tnw.tudelft.nl. determined by the speed with which the thermal energy can be removed from the microbridge. Based on the ratio between the thermal length $\lambda_{t h}=\sqrt{D \tau_{e-p h}}$ ( $D$ is the electronic diffusion constant and $\tau_{e-p h}$ is the electron-phonon coupling time) and the length of the microbridge $L_{b}$, HEBs can be divided into two classes.

If $L_{b} / \lambda_{t h}>1$, the hot electrons will primarily lose their energy via inelastic electron-phonon scattering within the microbridge. In this case one speaks of a phonon-cooled HEB [1]. Based on an electron-phonon coupling time of about $12 \mathrm{ps}$ for a thin $\mathrm{NbN}$ film, the IF bandwidth can be $10 \mathrm{GHz}$ which is large enough for practical applications [9]. Due to relatively long phonon escape time of $40 \mathrm{ps}$, the IF bandwidth is limited to $3-4 \mathrm{GHz}$ in practice. Recently, significant improvement of the gain bandwidth was achieved by using $\mathrm{MgO}$ as a substrate for the thin $\mathrm{NbN}$ film $\left(f_{-3 d B}=4.6 \mathrm{GHz}\right)$. Further optimization is expected to yield an IF bandwidth of $5-6 \mathrm{GHz}[10]$.

If, on the other hand, $L_{b} / \lambda_{t h}<1$, the heat transfer in the microbridge will be dominated by outdiffusion of hot electrons to the contacts. The idea of using diffusion-cooling rather than phonon-cooling to achieve a large bandwidth was proposed by Prober in 1993 [2]. Experimentally, the crossover from phonon-cooling to diffusion-cooling has been demonstrated by varying the length of the microbridge [11], [12]. Obviously, if the mixer is made very short, the IF rolloff frequency can be very high. Recently, an IF bandwidth of $9.2 \mathrm{GHz}$ was reported for a $100 \mathrm{~nm}$ long $\mathrm{Nb}$ microbridge, whereas a $80 \mathrm{~nm}$ long device showed no clear roll-off up to $15 \mathrm{GHz}[13]$.

We now consider the the time constant of a diffusioncooled superconducting HEB mixer in a bit more detail. For a diffusion-cooled metallic microbridge, the thermal relaxation time is given by [5], [14]

$$
\tau_{d i f}=\frac{L_{b}^{2}}{\pi^{2} D}
$$

This expression does take into account the presence of a temperature profile, but assumes no spatial variation of the diffusion constant, being determined by the (local) ratio of the thermal conductivity $K$ and the electronic heat capacity $c_{e}$. In practice, however, the rf pumped and $\mathrm{dc}$ biased HEB is cooled well below $T_{c}$, and operates in a electronic hotspot state. In this case, the center of the bridge is normal (hotspot region), whereas the ends of the microbridge are superconducting. In the normal region, the diffusion constant is independent of the local temperature, because both $K$ and $c_{e}$ have a linear temperature dependence. In the superconducting parts, however, $K$ decreases stronger with decreasing temperature $\left(K \sim T^{2} e^{-1.76 T_{c} / T}\right)$, 


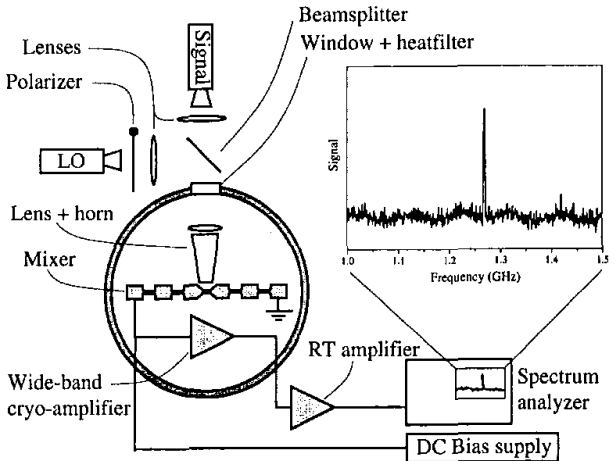

Fig. 1. Schematic representation of the experimental setup used for the gain vs. intemediate frequency measurement. The inset shows an actual measured spectrum

whereas the electronic heat capacity $c_{e}$ will be larger than in the normal part of the microbridge [15]. For simplicity, we assume that the ratio of $K / c_{e}$ can also be taken as the effective diffusion constant in the superconducting domains ${ }^{1}$, and, consequently, its value will be considerably lower in the superconducting regions. The average diffusion constant along the microbridge will therefore decrease with decreasing length of the hotspot. As a result, the time constant of the microbridge is expected to increase with decreasing length of hotspot, or, in order words, with decreasing bias voltage.

In this paper, this hypothesis is verified. We present the experimental results of the measurement of the IF bandwidth of a $\mathrm{Nb}$ diffusion-cooled HEB for different bias voltages i.e for different lengths of the electronic hotspot. The measured time constants are corrected for electro-thermal feedback through the IF circuit using the EHS model, in order to infer the intrinsic time constant of the mixer. The time constants are compared with the calculated time constant on the basis of a qualitative model.

\section{Measurements}

The IF bandwidth measurements have been performed around $700 \mathrm{GHz}$ in an experimental configuration which uses two local oscillators. The fabrication process of the device has been described in detail [17]. The device used in this experiment has a length of $300 \mathrm{~nm}$, a width of 200 $\mathrm{nm}$, and a normal state resistance $R_{n}$ of $53 \Omega$. The critical temperature $T_{c}$ of the microbridge is $5.9 \mathrm{~K}$. The diffusion constant was independently determined to be $1.6 \mathrm{~cm}^{2} / \mathrm{s}[4]$. The mixer is mounted in a tunable waveguide, designed for

${ }^{1}$ On a microscopic scale, the normal state diffusion constant is given by $v_{f} l_{e} / 3$, with $v_{f}$ the Fermi velocity of the electrons and $l_{e}$ the electron mean free path. In the superconducting state, the quasiparticles do no travel with the Fermi-velocity, their average velocity $\left\langle v_{q p}\right\rangle$ is substantially lower, and given by $\left\langle v_{q p}\right\rangle=v_{f}\left(\frac{2 k_{b} T}{\pi \Delta}\right)$, with $\Delta$ the energy gap of the superconductor. Since the inelastic scattering length for quasi-particles remains unchanged with respect to the normal state, this implies that the effictive diffusion constant in the superconducting parts of the microbridge is lower than its normal state value. (a)

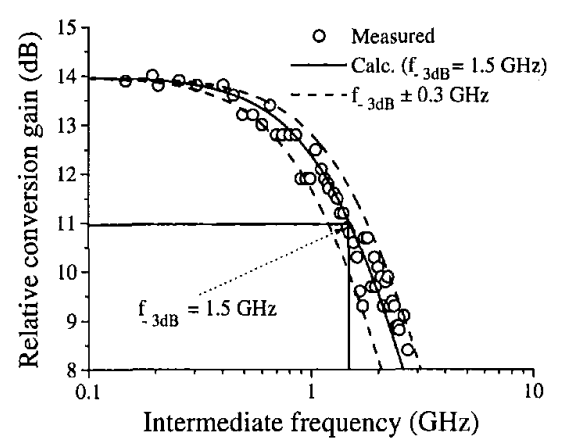

(b)

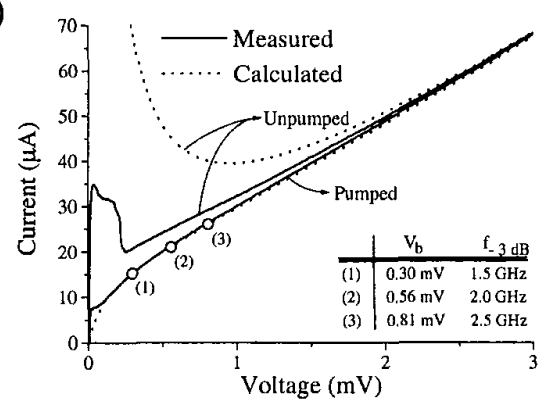

Fig. 2. (a) Relative conversion gain as a function of the intermediate frequency. The solid line is a one-pole calculation with a rolloff frequency $f_{-3 B}$ of $1.5 \mathrm{GHz}$. The dashed lines represent the error margins $(1.5 \pm 0.3 \mathrm{GHz})$. (b) Unpumped and pumped I(V) curves. The bias points where the IF bandwidth has been measured are indicated by the open squares. Also shown are the calculated I(V) curves based on the EHS model. The coupled LO power in the calculation is $37 \mathrm{nW}$.

frequencies around $700 \mathrm{GHz}$. The rf power to pump the device is provided by a carcinotron with a doubler, whereas a Gunn oscillator with a quadrupler acts as a (weak) signal source. The IF output signal is amplified by a $0.1-8 \mathrm{GHz}$ Miteq cryo-amplifier with an effective input impedance $R_{L}$ of $32 \Omega$ [17] and a $6 \mathrm{~dB}$ attenuator at the input to isolate the device from reflections. A $0.1-14 \mathrm{GHz}$ Miteq amplifier is used to amplify the signal at room temperature. The amplified signal is measured with a spectrum analyzer. Special attention is paid to the gain versus frequency calibration of the IF chain and saturation of the second amplifier. During the experiment, the signal frequency is kept constant and the LO frequency is varied. The LO power is kept constant by adjusting the polarizer in the optical path and maintaining a constant current through the device with an accuracy better than $1 \mu \mathrm{A}$. The bath temperature during the experiment is $4.5 \mathrm{~K}$. Fig. 1 shows a schematic representation of the experimental setup.

The measured relative conversion gain as a function of the intermediate frequency at $V_{b}=0.30 \mathrm{mV}$ is shown in Fig. 2a. The calculated bandwidth from a one-pole fit to the data is $1.5 \mathrm{GHz}$, corresponding to an effective thermal time of $\tau_{e f f}=\left(2 \pi f_{-3 d B}\right)^{-1}=106 \mathrm{ps}$. The maximum error in the measurement is $0.3 \mathrm{GHz}$ and the corresponding curves with a roll-off frequency $f_{-3 d B}$ of $1.2 \mathrm{GHz}$ and $1.8 \mathrm{GHz}$ 
are also plotted in Fig. 2a. Fig. 2b shows the unpumped and pumped $I(V)$ curves of the device. The three bias points at which the IF bandwidth has been measured are indicated in the figure, and the corresponding values for the bandwidth are given in the table in the inset. Clearly, the IF bandwidth increases with increasing bias voltage, up to $2.5 \mathrm{GHz}$ at $V_{b}=0.81 \mathrm{mV}$. The similar effect was observed by Skalare et al. on a comparable device, but the physical origin of the effect was not addressed [18]. Furthermore, it should be interesting to know what is the value of $f_{-3 d B}$ expected from Eq. 1. We find $\tau_{d i f}$ to be $57 \mathrm{ps}$, giving an $f_{-3 d B}$ of $2.8 \mathrm{GHz}$.

\section{Data Analysis}

\section{A. Selfheating through the IF load in the hotspot framework}

In order to understand our experimental results and infer the intrinsic time constant of the mixer, the data have to be corrected for electro-thermal feedback through the IF load [3], [19]. The measured effective time constant $\tau_{\text {eff }}$ of a $\mathrm{HEB}$ mixer is related to the intrinsic time constant $\tau_{t h}$ via

$$
\tau_{e f f}(V)=\frac{\tau_{t h}}{1+\alpha(V) \beta(V)}
$$

The factors $\alpha(V)$ and $\beta(V)$ can be derived from the pumped $I(V)$ characteristic and are given by

$$
\alpha(V)=\frac{I_{d c}^{2}\left(\frac{d R}{d T}\right)}{G}, \beta(V)=\frac{R_{d c}-R_{L}}{R_{d c}+R_{L}},
$$

with $G$ the thermal conductance, and $R$ the dc resistance $V / I$ at the bias-point of interest. The factor $\alpha(V)$ reflects the selfheating of the device, whereas $\beta(V)$ accounts for the finite impedance of the IF load, basically suppressing the effect of electro-thermal feedback. In the lumped element approach, $\alpha(V)$ can be calculated directly from the pumped $I(V)$ curve [19]. In the hotspot framework, however, a temperature profile is present, and the same change in $\mathrm{rf}$ and dc heating has a different effect on the resistance of the HEB [6], [7]. Therefore, $(\mathrm{dR} / \mathrm{d} \mathrm{T}) G^{-1}$ should be rewritten as

$$
\left(\frac{d R}{d T}\right) G^{-1}=\frac{C_{r f} G_{r f}+C_{d c} G_{d c}}{G_{r f}+G_{d c}}
$$

with

$$
C_{r f, d c}=\left(\frac{\partial R}{\partial P_{r f, d c}}\right), G_{r f, d c}=\left(\frac{\partial \vec{T}}{\partial P_{r f, d c}}\right)^{-1}
$$

Here, $C_{r f, d c}$ reflects the change in resistance due to a change in dissipated $\mathrm{rf}$ or dc power, respectively, $G_{r f, d c}$ is the effective thermal conductance for rf or dc heating, and $\bar{T}$ is defined as the average temperature along the microbridge. Both $C_{r f, d c}$ and $G_{r f, d c}$ can be calculated using the appropriate heat balance equations for a HEB. Note that in the case that $C_{r f}=C_{d c}$ and $G_{r f}=G_{d c}$, Eq. 4 reduces to the expression given by [3],[19].
TABLE I

OVERVIEW OF THE RESULTS OF THE EXPERIMENT AND ANALYSIS FOR THE THREE BIAS POINTS.

\begin{tabular}{cccccc}
\hline \hline$V_{b}(m V)$ & $L_{h}(n m)$ & $\tau_{\text {eff }}(p s)$ & $\tau_{t h}(p s)$ & $\alpha(V)$ & $\beta(V)$ \\
\hline 0.30 & 113 & 106 & 100 & 0.28 & -0.21 \\
0.56 & 148 & 80 & 79 & 0.26 & -0.058 \\
0.81 & 173 & 64 & 64 & 0.23 & 0.021 \\
\hline \hline
\end{tabular}

$\overline{V_{b}}$ is the bias voltage, $\bar{L}_{h}$ the length of the hotspot, $\tau_{e f f}$ the mea-

sured effective time constant, and $\tau_{t h}$ the intrinsic time constant. The factors $\alpha(V)$ and $\beta(V)$ are used to correct $\tau_{e f f}$ for electro-thermal feedback.

\section{B. Electro-thermal analysis of the mixer}

We now proceed by modeling the electro-thermal behaviour of the HEB mixer using the physically justified electronic hotspot model [6]. Here, we introduce two modifications with respect to the model presented in chapter IV, which physically describe a diffusion-cooled HEB more accurately, although they are found to have little influence on the actual outcome of the calculations. First, we do not take the thermal conductivity $K$ to be constant but assume that it is linearly increasing with temperature in both normal and superconducting parts according to the Wiedemann-Franz law. Second, we neglect the heat transfer from electrons to phonons, justifiable for a diffusioncooled HEB, and allowing an analytical solution to the problem. As a result, the heat balance equation for a biased and pumped diffusion-cooled HEB takes the following form: Inside the hotspot, we have

$$
-\frac{d}{d x}\left(K(T) \frac{d T}{d x}\right)=j^{2} \rho_{n}+p_{r f}
$$

with $j$ the dc current density in the microbridge and $p_{r f}$ the rf power density (per unit volume). Outside the hotspot we use the same equation, with the exception that there the dc dissipation is zero $\left(j^{2} \rho_{n} \rightarrow 0\right)$. In the analysis, it is assumed that rf power is absorbed homogeneously in the microbridge, which is true if the frequency of the radiation is above the gap frequency of the superconductor. In our case, the radiation frequency is $700 \mathrm{GHz}$, whereas the gap frequency is $\approx 450 \mathrm{GHz}$. The equations are solved by requiring that $T=T_{b}$ at the end of the bridge and $T=T_{c}$ at the hotspot boundary. The current required to sustain a hotspot of length $L_{h}$ when a radiation power density $p_{r f}$ is absorbed, follows from matching $d T / d x$ at the hotspot boundary.

In Fig. 2b, we plot the calculated pumped and unpumped $I(V)$ characteristics together with the experimental data. A good agreement is found between measurement and calculation in the case of the pumped $I(V)$ curve. In the case of the unpumped characteristic, deviations between model and measurement are observed at low bias voltages, which show that the measured minimum dc current needed to sustain a hotspot is smaller than predicted by the model. This observation has been discussed in more detail [20]. 


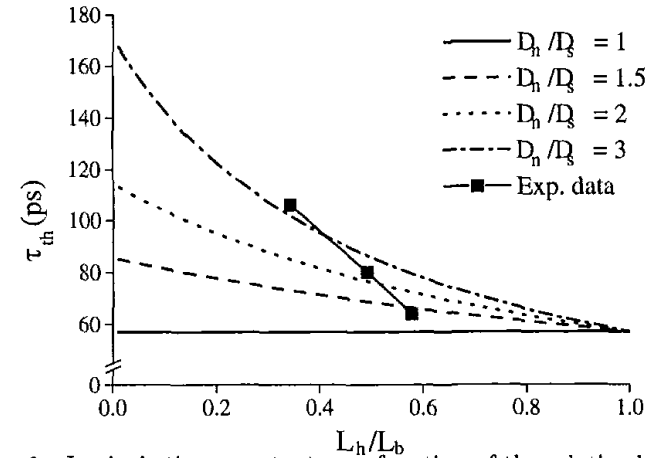

Fig. 3. Intrinsic time constant as a function of the relative length of the hotspot. 'The squares indicate the experimental results. The curves are calculated using different values of the ratio $D_{n} / D_{s}$ $\left(D_{n}=1.6 \mathrm{~cm}^{2} / \mathrm{s}, L_{b}=300 \mathrm{~nm}\right)$.

We also calculate the temperature profile of the electrons within the bridge for different rf and dc powers. Using eqs. $2-5$, we can determine values of $\alpha(V)$ and $\beta(V)$, and eventually derive $\tau_{t h}$. The lengths of the hotspot for the three bias points are calculated on the basis of the dc resistance $R_{d c}$ in according to $L_{h}=\left(R / R_{n}\right) L_{b}$. In Table $I$, the results of the measurement and calculation for the three different bias points are summarized, from which it becomes clear that the intrinsic time constant of the microbridge increases with increasing length of the hotspot. Note that in our particular case the effect of electro-thermal feedback is largely suppressed by the IF circuit, because the impedances of the IF load and mixer are close to each other. As a result, the effective and intrinsic thermal times do not differ much.

\section{Discussion and Conclusion}

In order to verify whether the outcome of the experiment is physically sensible, we compare the results with a calculation of the time constant on the basis of Eq. 1, taking into account the difference in the effective diffusion constants of the normal and superconducting parts of the microbridge. We assume that we can use a linearly weighted average value of the diffusion constant according to

$$
\bar{D}\left(L_{h}\right)=\frac{L_{h} D_{n}+\left(L_{b}-L_{h}\right) D_{s}}{L_{b}}
$$

with $D_{n}$ and $D_{s}$ the effective diffusion constant in the normal and superconducting regions, respectively. In Fig. 3, we plot the calculated time constant for different (realistic) values of $D_{n} / D_{s}$ as a function of $L_{h} / L_{b}$. Here we do not choose a fixed ratio of $D_{n} / D_{s}$ simply because there is a temperature profile within the bridge. This profile depends also on the bias voltage (dc heating power). So, the exact ratio is difficult to know. As indicated by Fig. 3 , the predicted trend corresponds to the experimentally observed one. However, we do not obtain nor expect a one-to-one correspondence between measurement and calculation, because the model used is oversimplified. A more realistic calculation would have to include the full time- dependent heat-balance equations, taking into account the bias-dependent modification of the time constant due to electro-thermal feedback. Moreover, we should recognize that in using Eq. 7, we follow a phenomenological approach, which ignores the possible energy dependences of the thermal transport processes.

To conclude, we demonstrate that the intrinsic time constant of a diffusion-cooled HEB depends not only on the length of the microbridge, but also on length of the hotspot in the bridge, the latter being determined by the dc bias voltage and applied rf pumping power. The observations can be understood by using a qualitative model in which the reduced effective diffusion constant in the superconducting regions is taken into account

\section{REFERENCES}

[1] E.M. Gershenzon, G.N. Gol'tsman, I.G. Gogidze, Y.P. Gusev, A.I. Elantiev, B.S. Karasik, and A.D. Semenov, Sov. Phys. Superconductivity, vol. 63 , pp. 1582, 1990 .

[2] D.E. Prober, Appl. Phys. Lett., vol. 62, pp. 2119, 1993.

[3] B.S. Karasik and A.I. Elantiev, Appl. Phys. Lett. vol. 68, pp. 853, 1996.

[4] D. Wilms Floet, J.J.A. Baselmans, T.M. Klapwijk, and J.R. Gao, Appl. Phys. Lett., vol. 73, pp. 2826, 1998.

[5] P. J. Burke, Ph. D. thesis, Yale University, 1997.

[6] D. Wilms Floet, E. Miedema, T.M. Klapwijk, and J.R. Gao, Appl. Phys. Lett., vol. 74, pp. 433, 1999.

[7] H. Merkel, P. Khosropanah, P. Yagoubov, and E. Kollberg, Proceedings Tenth International Symposium Space Terahertz Technology, University of Virginia, Charlottesville, pp. 592-606, March 16-18, 1999.

[8] A. Skalare, W.R. McGrath, B. Bumble, H.G. LeDuc, Proceedings Tenth International Symposium Space Terahertz Technology, University of Virginia, Charlottesville, pp. 539-551, March 16-18, 1999.

[9] Y.P. Gousev, G.N. Gol'tsman, A.D. Semenov, and E.M. Gershenzon, R.S. Nebosis, M.A. Heusinger, and K.F. Renk, J. Appl. Phys., vol. 75, pp. 3695, 1994.

[10] S. Cherednichenko, M. Kroug, P. Yagoubov, H. Merkel, E. Kollberg, K.S. Yngvesson, B. Voronov, and G. Gol'tsman, Presented at the Eleventh International Symposium Space Terahertz Technology, University of Michigan, USA, May 1-3, 2000.

[11] P.J. Burke, R.J. Schoelkopf, D.E. Prober, A. Skalare, W.R. McGrath, B. Bumble, and H.G. LeDuc, Appl. Phys. Lett., vol. 68, pp. 3344, 1996.

[12] B.S. Karasik, K.S. Il'in, E.V. Pechen, and S.I. Krasnosvobodtsev, Appl. Phys. Lett., vol. 68, pp. 2285, 1996.

[13] R.A. Wyss, B.S. Karasik, W.R. McGrath, B. Bumble, and H. LeDuc, Proceedings of the Tenth International Symposium Space Terahertz Technology, University of Virginia, Charlottesville, pp. 215-228, March 16-18, 1999.

[14] H. Carslaw and J. Jeager, Conduction of Heat in Solids, Oxford University Press, London, 1959.

[15] F. Pobell, Matter and methods at low temperatures, Berlin, Springer-Verlag, 1992.

[16] D. Wilms Floet, J.R. Gao, W. Hulshoff, H. van de Stadt, T.M. Klapwijk, and A.K. Suurling, IOP Conf. Series 158 , edited by H. Rogalla and D.H.A. Blank, pp. 401, 1997.

[17] Based on specifications with respect to the voltage standing wave ratio of the amplifier at room temperature by Miteq Inc., 100 Davids Drive, Hauppauge, N.Y. 11788.

[18] A. Skalare, W.R. McGrath, B. Bumble, H.G. LeDuc, P.J. Burke, A.A. Verheijen, R.J. Schoelkopf, and D.E. Prober, Appl. Phys. Lett., vol. 68 , pp. $1558,1996$.

[19] H. Ekström, B.S. Karasik, E. Kollberg, and K.S. Yngvesson, IEEE Trans. Microwave Theory Tech., vol. 43, pp. 938, 1995.

[20] D. Wilms Floet, J.R. Gao, T.M. Klapwijk, and P.A.J. de Korte, Proceedings Tenth International Symposium Space Terahertz Technology, University of Virginia, Charlottesville, pp. 538 591, March 16-18, 1999. 